\title{
Un enfoque inferencial y dinámico de la modelización y la comprensión en biología
}

\author{
An inferential and dynamic approach to modeling and \\ understanding in biology
}

\author{
Rodrigo Lopez-Orellana*; Juan Redmond**; David Cortés-García ${ }^{\dagger}$ \\ *CONICYT, Chile / Instituto de Estudios de la Ciencia y la Tecnología, \\ Universidad de Salamanca, España \\ rodrigo.lopez@usal.es \\ ${ }^{* *}$ Universidad de Valparaíso, Chile \\ juan.redmond@uv.cl \\ Universidad del País Vasco, España \\ davidcortesgarcia.dcg@gmail.com
}

\begin{abstract}
Resumen
El objetivo del presente trabajo es proponer un enfoque inferencial y dinámico de la comprensión con modelos en biología. La comprensión cumple un papel central en la práctica de la modelización. A partir de su vinculación con los otros dos elementos centrales de la investigación científica, experimentación y explicación, mostramos su relevancia epistémica para el caso de la explicación en biología. Además, gracias a la introducción de la noción de comprensión, proponemos una perspectiva no referencialista de los modelos científicos, que está determinada por su uso.
\end{abstract}

Palabras clave: comprensión, modelos, explicación, representación, experimentación, inferencias, razonamiento subrogativo, biología.

\section{Abstract}

This paper aims to propose an inferential and dynamic approach to understanding with models in biology. Understanding plays a central role in the practice of modeling. From its links with the other two central elements of scientific research, experimentation,

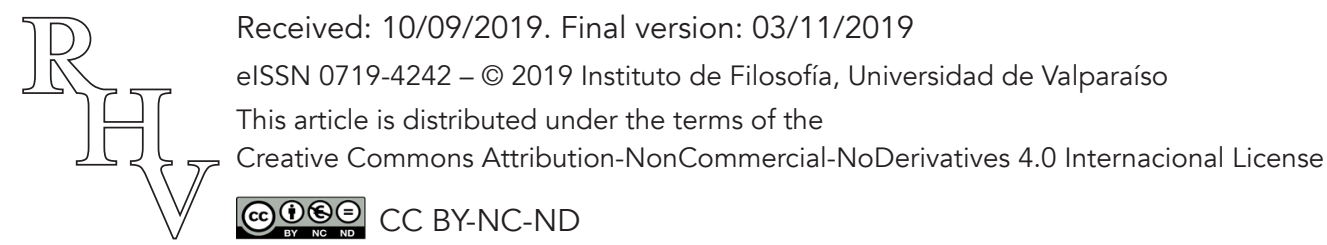


Un enfoque inferencial y dinámico de la modelización y de la comprensión en biología

Rodrigo Lopez-Orellana; Juan Redmond; David Cortés-García

and explanation, we show its epistemic relevance to the case of explanation in biology. Furthermore, by including the notion of understanding, we propose a non-referentialist perspective on scientific models, which is determined by their use.

Keywords: understanding, models, explanation, representation, experimentation, inferences, surrogate reasoning, biology.

\section{Introducción}

En filosofía de la ciencia se ha argumentado que los modelos científicos cumplen dos funciones que no son excluyentes: 1) representan una porción de la realidad (sistema-dia$n a$; sistema de fenómenos); $\mathrm{y}, 2$ ) representan una teoría en el sentido de que interpretan las leyes o axiomas de esta (Frigg y Nguyen 2016; Frigg y Hartmann 2018). Es en el primer sentido, principalmente, que la práctica de modelización en nuestros días tiene un gran protagonismo en la literatura filosófica. En este artículo ofrecemos algunos elementos de discusión al respecto, centrándonos en el punto 1) dentro del ámbito de la biología: nuestro objetivo general es profundizar en cómo los modelos representan sus sistemas-diana (target-systems) de tal modo que la práctica de modelar se ajuste a la evidencia científica.

Nuestro enfoque está alineado con las perspectivas que critican la suposición de que la representación es una relación diádica de correspondencia entre el vehículo representativo (el modelo) y su sistema-diana (v. g., Cartwright, Shomar y Suárez 1995; Morrison y Morgan 1999; Suárez 2003; Suárez 2004; Knuuttila y Merz 2009). Por ello, nos distanciamos de cualquier noción de representación como 'espejo', 'especulo' o 'replicación' (mirroring), que tienen que ver con conceptos como isomorfismo, similitud (likeness) o semejanza (resemblance) entre las propiedades del vehículo de representación y las de su sistema-diana. Nada parece más alejado de esto último que la práctica misma de la modelización en biología.

Uno de los objetivos principales que trata de satisfacer la modelización científica es proporcionar explicaciones acerca de los fenómenos que representan. En términos generales, se entiende por 'explicación científica' la acción o el proceso de establecer y especificar relaciones (causales, funcionales, mecanicistas, históricas, etc.) entre fenómenos, principalmente a través de teorías científicas. Sin embargo, en el caso concreto de la biología son los modelos los que son usados como las herramientas explicativas preferidas: "la explicación en biología se obtiene siempre a través de la intervención directa sobre modelos del fenómeno a explicar" (Leonelli 2009, 192). La característica singular de la biología es que explica esencialmente con modelos. Distinto es el caso de la física o la química donde los modelos comparten ese rol junto con las leyes científicas (en sentido propio; Lorenzano 2008), porque se insiste en las relaciones fenoménicas de tipo causal. En biología, explicación y representación están fuertemente entrelazadas.

Revista de Humanidades de Valparaíso, 2019, No 14, 315-334

(c) $(1)(9)$ CC BY-NC-ND 
Un enfoque inferencial y dinámico de la modelización y de la comprensión en biología

Rodrigo Lopez-Orellana; Juan Redmond; David Cortés-García

En los últimos años ha existido una rica discusión filosófica acerca de las funciones representacional y explicativa de los modelos. Por ejemplo, según Cassini $(2016,25)$, uno de los aspectos más interesantes de esta discusión es que - en general - los modelos científicos no siempre comportan un mecanismo causal para la producción de los fenó$\operatorname{menos}^{1}$ que se intentan representar. Aunque la mayoría de los modelos no son causales, sí muchos de ellos logran proporcionar explicaciones satisfactorias. Por esta razón, la discusión filosófica se ha ampliado para considerar otros tipos de explicaciones científicas que pueden ser aceptadas como legítimas (v. g., Woodward 2003; Kitcher 1989, 1981). De esta manera, el problema no queda reducido a las explicaciones causales - en un sentido fuerte-, especialmente para el caso de la biología. En esta ciencia abundan explicaciones funcionales, mecanicistas y también históricas-evolutivas. Aunque las más estudiadas por los filósofos de la biología han sido las explicaciones funcionales. Según Braillard y Malaterre $(2015,13)$, este tipo de explicaciones son problemáticas porque implican la noción de fin y por lo tanto de teleología, nociones que atraviesan tanto las explicaciones de la estructura de los organismos y de sus rasgos, como de sus características y organización. Además, la misma noción de función es muy compleja, se involucra en aquellas explicaciones que no tratan de dar cuenta de relaciones causa-efecto, sino que pretenden explicar las relaciones entre elementos como, por ejemplo, las ventajas asociadas a la aparición de un determinado carácter fenotípico en un contexto histórico y ambiental particular. Sin embargo, en biología sí es posible capturar con modelos este tipo de relaciones.

Junto con esto, cabe preguntarse también si en biología es necesario ampliar la perspectiva de la noción de representación o, más específicamente, preguntarse por cuál ha de ser la noción de representación estrictamente involucrada. Una forma de comenzar sería revisando el aspecto de intencionalidad que comporta la modelización, tal como se expresa en la cita anterior de Leonelli (2009), que se traduce en el uso que un agente (el científico) hace de un modelo para realizar una acción determinada sobre los fenómenos. En efecto, el componente intencional apremia a dirigir el análisis de los modelos hacia el estudio de las propias prácticas científicas: en cómo los biólogos realizan su investigación (experimentación) y construyen y utilizan modelos para representar o explicar los fenómenos.

En este trabajo ofrecemos entonces 1) algunos argumentos a favor de ampliar la perspectiva de la explicación con modelos incorporando la noción de comprensión, que actualmente se ha vuelto de gran interés para la filosofía de las ciencias naturales en el debate acerca de la modelización científica (de Regt y Dieks 2005; de Regt et al. 2009). Sin duda,

\footnotetext{
${ }^{1}$ Entiéndase por 'producción de fenómenos' la generación, creación o recreación de fenómenos a través de técnicas o procedimientos científicos, generalmente dentro de un laboratorio, para su estudio o descripción. Esta producción se da en el experimento científico, entendido como "un conjunto de dispositivos, mecanismos, sistema de métodos que mezclan sustancias con máquinas, seres vivos producidos por cría selectiva [...]" (Santesmases 2002, 267), entre otros elementos, y que se usan con el propósito de producir fenómenos en un laboratorio como fenómenos naturales.
}

Revista de Humanidades de Valparaíso, 2019, No 14, 315-334 
Un enfoque inferencial y dinámico de la modelización y de la comprensión en biología

Rodrigo Lopez-Orellana; Juan Redmond; David Cortés-García

esta noción ofrece una forma interesante de abordar el componente intencional de la modelización científica. En efecto, sostenemos que la comprensión tiene una relevancia epistémica para el caso de la explicación con modelos en biología. Además, gracias a la introducción de la noción de comprensión, 2) proponemos una perspectiva no referencialista sobre la modelización científica, determinada por el uso de modelos, la experimentación y la producción de fenómenos.

Pero antes de exponer nuestro enfoque, conviene tener presente lo siguiente. Frigg y Nguyen (2016) argumentan que - en términos generales - toda perspectiva sobre la representación científica, que pretenda estar bien definida, debe dar cuenta de los siguientes ítems:

(1) El problema de la demarcación representacional: la pregunta acerca de cómo las representaciones científicas difieren de otros tipos de representaciones (criterios de representación científica).

(2) Si el propósito que se tiene es establecer la demarcación entre representaciones científicas y las que no lo son, entonces se debe abordar el problema de la representación cientifica: cómo llenar el espacio en blanco en ' $R$ es una representación científica del sistema-diana $S D$ sys __'2. En cambio, si se parte del rechazo al problema de la demarcación representacional, tendrá que abordarse el problema de la representación epistémica: cómo llenar el espacio en ' $R$ es una representación epistémica de $S D$ sys ' (ER-Scheme).

(3) El problema de estilo: ¿qué estilos de representación existen y cómo se pueden caracterizar?

(4) Formular estándares de precisión (accuracy): ¿cómo identificamos lo que constituye una representación precisa o fiable (an accurate representation)?

(5) El problema ontológico: ¿cuáles son los tipos de objetos que sirven como representación?

Además, Frigg y Nguyen nos dicen que junto con estos problemas también tenemos las siguientes condiciones de adecuación o de suficiencia, que deben cumplirse satisfactoriamente:

(6) Razonamiento subrogativo (surrogate reasoning): las representaciones científicas nos permiten generar hipótesis sobre sus sistemas-diana.

\footnotetext{
${ }^{2}$ Hemos cambiado ' $S$ ' (representation) por ' $R$ ', y también ' $T$ ' (target system) por ' $S D$ ' (sistema-diana), del esquema original de Frigg y Nguyen (2016).
} 
Un enfoque inferencial y dinámico de la modelización y de la comprensión en biología

Rodrigo Lopez-Orellana; Juan Redmond; David Cortés-García

(7) Posibilidad de distorsión (misrepresentation): si $R$ no representa exactamente a $S D$, entonces se trata de una distorsión pero no de una no-representación (Redmond et al. 2017).

(8) Modelos sin diana (targetless): dar respuesta a la pregunta ¿qué debemos hacer con las representaciones científicas que carecen de targets, pero que son exitosas de igual manera?

(9) Requisito de direccionalidad: las representaciones científicas se refieren a sus targets, pero los targets no se refieren a sus representaciones.

(10) Aplicabilidad de las matemáticas: ¿cómo el aparato matemático utilizado en algunas representaciones científicas se vincula o enlaza con el mundo físico?

En nuestro artículo solo nos focalizaremos en aquellos aspectos y condiciones que consideramos más determinantes para la práctica de modelización en biología. Especialmente aquellos aspectos y condiciones que se orientan a proponer una nueva definición de representación incluyendo la noción de comprensión. Es en este sentido que nos ocuparemos del problema de la representación (2) y lo resolveremos en términos inferenciales desde el razonamiento subrogativo (6) como condición de suficiencia. Ejemplificaremos nuestro punto, muy brevemente, a partir de aplicaciones matemáticas (10) en modelos metapoblacionales.

\section{Comprensión científica a partir de modelos}

En primer lugar, afirmamos que la comprensión — en términos generales - debe entenderse como un estado subjetivo en el cual se construyen significados y/o conocimientos acerca de hechos o ideas y que pertenece a un dominio cognitivo como pensamiento inferencial. La comprensión permite de una manera fiable organizar, interpretar, comparar, traducir, describir la información o sacar conclusiones generales o resolver problemas a partir de ella. Por este motivo, no solo es un estado, sino que también es una acción. Pero esta acción no es reducible a tipos de razonamiento deductivos o inductivos, sino que se configura por medio de un tipo de disposiciones y relaciones de distinto carácter ${ }^{3}$.

En el caso del razonamiento científico, la información involucrada en la comprensión es el resultado de la investigación, teorización y experimentación científica. En este caso podemos distinguir entre comprensión y explicación científicas en cuanto a que la primera es una acción subjetiva, de un agente (el científico), aunque basada en una información acerca de los fenómenos del mundo (como información relevante, no trivial) obtenida a partir de la propia práctica científica. En cambio, la segunda siempre es intersubjetiva:

\footnotetext{
${ }^{3}$ Para un análisis acerca del razonamiento subrogativo, especialmente sobre los problemas de utilizar lógica clásica en este tipo de razonamiento, véase Redmond y Lopez-Orellana (2018a).
}

Revista de Humanidades de Valparaíso, 2019, No 14, 315-334 
Un enfoque inferencial y dinámico de la modelización y de la comprensión en biología

Rodrigo Lopez-Orellana; Juan Redmond; David Cortés-García

es llevada a cabo por un agente pero involucra de forma directa el reconocimiento de su éxito y aceptación por parte de una comunidad de agentes (la comunidad científica), en relación con ciertos procedimientos y criterios que esa comunidad ha establecido explícitamente. Por ello, la explicación científica ha estado fuertemente relacionada a tipos de razonamiento deductivos, inductivos, probabilísticos o, por lo menos, de acuerdo con ciertas reglas generalmente explícitas y aceptadas por dicha comunidad.

Según de Regt et al. $(2009,7)$, en la filosofía de la ciencia la comprensión se ha visto como un subproducto de la explicación, relegada a un segundo plano. Creemos que esto se debe, por un lado, a la necesidad de evitar consecuencias innecesarias de hipótesis psicologistas sobre el conocimiento científico; y, por otro lado, se debe a la exagerada importancia que se le ha dado al papel de las teorías y las leyes. Hoy en día, distintos autores limitan ese papel de las teorías a su utilidad como instrucciones para la construcción de modelos (principalmente de modelos explicativos) y ponen el foco en cuestiones tales como la autonomía de los modelos (respecto de las teorías), su manipulabilidad o sus usos, la producción de conocimiento y de fenómenos, o la comprensión acerca de los fenómenos que se gana con su uso (Knuuttila y Merz 2009, 153-154).

En la práctica científica es posible reconocer una variedad de relaciones entre sujeto cognoscente y fenómenos que caben bajo la forma de la comprensión (Wilkenfeld 2013, 999), razón por la cual la elaboración de una explicación 'unificada' de la comprensión científica puede resultar un tanto difícil. Sin embargo, de ello no se sigue que la noción de comprensión carezca de relevancia epistémica y que no pueda darse una definición más o menos general. En efecto, un esfuerzo de caracterización general dentro del debate de la explicación lo realizan de Regt et al. (2005; 2009), a partir de un criterio esencialmente contextual para la inteligibilidad de las teorías científicas.

Respecto a la cuestión de la relevancia epistémica, sabemos que los modelos pueden ofrecer una mejor comprensión de los fenómenos. Como argumenta Newman $(2017,574)$, dicha comprensión en ningún caso es modesta o superficial. Debemos reconocer que en la comprensión se garantiza - de alguna manera - un logro cognitivo efectivo: 'el sujeto $S$ comprende un sistema de fenómeno $f$ por medio del modelo $M$ '. Si esto no fuera así, es decir, si $S$ comprendiera $f$ solo de una manera superficial, observaríamos — por ejemploque $S$ no sería capaz de usar $M$ para ejecutar acciones o resolver problemas relativos al estudio de $f$. Pero, como argumenta Newman, sí vemos que en la propia práctica de la modelización - $\mathrm{y}$, sobre todo, de la experimentación - los científicos son capaces de resolver esos problemas o ejecutar acciones significativas; incluso, evaluar la plausibilidad de $M$ o de la teoría $T$ involucrada, y ganar conocimiento respecto de $T$.

Una buena definición de la noción de comprensión ha sido elaborada por Elgin (2009, 327):

Revista de Humanidades de Valparaíso, 2019, No 14, 315-334

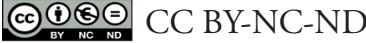


Un enfoque inferencial y dinámico de la modelización y de la comprensión en biología

Rodrigo Lopez-Orellana; Juan Redmond; David Cortés-García

La comprensión es la captación [grasp] de un cuerpo general y amplio de información que está basada en los hechos, que responde debidamente a la evidencia y nos capacita para realizar inferencias no triviales, argumentos, y quizás acciones relacionadas con el asunto al que la información pertenece. (Elgin 2009, 327, traducción nuestra)

Además, esta noción

[...] se aplica a la comprensión como el producto final de la actividad explicativa ${ }^{4}$ (el estado cognitivo alcanzado, el objetivo de la explicación), porque es el estado de un sujeto cognitivo. Y se aplica aún más a la comprensión de las teorías y modelos utilizados en estas actividades explicativas: tal comprensión se refiere a la utilidad y a la manipulabilidad de las teorías y modelos evaluadas por un grupo específico de pares. (de Regt et al. 2009, 3, traducción e itálicas nuestras)

Esta idea supone que la comprensión pertenece no solo a un dominio cognitivo, sino también al dominio de la pragmática: un análisis acerca de los modelos debe incluir cuestiones como los propósitos del científico y los recursos materiales y abstractos que emplea para conseguirlos.

Cabe advertir que no entraremos aquí en la discusión respecto a los términos 'captación' (grasp) e 'información', que son por sí mismos bastante problemáticos. Remitimos a los trabajos de Newman (2017), Elgin (2009) y de Regt et al. (2009). Sí insistiremos en la inclusión de las nociones de 'utilidad' (también 'uso') y 'manipulación' (usefulness and tractability) señaladas en la cita anterior. Para el caso que nos interesa, esto es, sobre el papel que ocupa la comprensión en biología, lo que se pone en juego es que la manipulación tiene un valor epistémico central. El ámbito primordial de esa manipulación es el experimento. El objetivo de esa manipulación es conseguir una explicación de los fenómenos. Pero el objeto de esa manipulación es un modelo. Leonelli (2009) entiende este tipo de manipulación de modelos como "intervención directa":

Por "intervención directa" me refiero a la modificación de un modelo para investigar el fenómeno biológico que ese modelo representa. Esto puede suceder a través del razonamiento, como cuando se cambian mentalmente los términos de una ecuación usada para representar la dinámica evolutiva, o a través de movimientos físicos, como cuando

\footnotetext{
${ }^{4}$ Debemos advertir que la comprensión no siempre resulta ser el producto final de la explicación. Podemos tener explicaciones científicas exitosas sobre los fenómenos y, sin embargo, no lograr comprensión alguna sobre esos fenómenos o sus detalles. Por ejemplo, Darwin admitía que, si bien su teoría era una explicación muy exitosa de la evolución biológica a través de la selección natural, no había logrado comprender del todo algunos de sus mecanismos (cf. Darwin 1859, 194-195, Capítulo VI, sobre las dificultades de su teoría). Obviamente, explicación y comprensión son dos actividades intelectuales muy distintas, pero que pueden complementarse. Juntas pueden producir una mayor ganancia de conocimiento, pero va a depender de cuestiones como la inteligibilidad de las teorías o de los modelos. Un análisis detallado al respecto puede encontrarse en de Regt (2009, 30-38).
}

Revista de Humanidades de Valparaíso, 2019, No 14, 315-334 
Un enfoque inferencial y dinámico de la modelización y de la comprensión en biología

Rodrigo Lopez-Orellana; Juan Redmond; David Cortés-García

se cambia el orden de las bolas que representan los nucleótidos en una representación plástica de la doble hélice del ADN. Ya sea que se exprese en pensamientos o gestos, la agencia humana constituye una condición ineludible para producir explicaciones del mundo natural. (Leonelli 2009, 192, traducción e itálicas nuestras)

Por tanto, el 'uso' de un modelo implica siempre una manipulación o intervención directa sobre los modelos de los fenómenos a explicar, y la comprensión juega un rol central en esta acción. En este sentido, "[1]a comprensión es un término de éxito cognitivo" (Elgin 2009, 327), ya que nos permite decir con propiedad algo acerca de los fenómenos e inferir nuevas consecuencias acerca de ellos, a la vez que nos permite actuar sobre los propios modelos y sus sistemas-diana. Este tipo de comprensión obedece al interés por parte del científico de ofrecer una información relevante y pertinente sobre los fenómenos estudiados y con la intención de que pueda ser considerada dentro del marco teórico general de una ciencia. En este proceso, los experimentos tienen un papel fundamental, como mostraremos más adelante.

\section{Enfoque inferencial y dinámico de la comprensión y del uso de los modelos}

El esquema que hemos visto anteriormente, 'el sujeto $S$ comprende un sistema de fenómenos $f$ pormedio del modelo $M$ ', puede traducirse de la siguiente manera (en un segundo nivel de comprensión):

El sujeto $S$ comprende $M$, que habla acerca de $f$, cuando $u s a M$.

Pero ¿qué estamos entendiendo por uso? Nuestra definición está inspirada en el enfoque inferencial presentado por Suárez (2004) y en la caracterización de Mäki (2009) acerca de la representación por sistemas subrogativos o sustitutos ${ }^{5}$ :

Decimos que un sujeto $S$ usa un modelo $M$ cuando:

(i) M apunta a un sistema-diana $S D$ (a un sistema de fenómenos); y

\footnotetext{
${ }^{5}$ Según a Mäki (2009, 32-33), debe entenderse que un modelo es un modelo de algo, específicamente de un sistema de fenómenos. Lo que, en estricto rigor, no es una perogrullada. Un modelo representa ese sistema de fenómenos como su sustituto. Un modelo $M$ representa un sistema-diana $S D$ en el sentido de que $M$ es representativo de $S D$ : en otras palabras, $M$ es el sistema sustituto/subrogante de $S D$. Para Mäki, la función epistémica de los modelos como sustitutos se traduce en conseguir conocimiento no examinando directamente a $S D$, sino más bien examinando directamente a $M$ (conocimiento indirecto de $S D$ ), siempre y cuando $M$ se parece o corresponde al sistema de destino $R$ en aspectos adecuados y en grados suficientes. Como hemos señalado anteriormente, en nuestro artículo analizamos cuál es la forma más ajustada a la evidencia científica en la que se relacionan $M$ y $S D$, como sistema de fenómenos biológicos, y cómo conseguimos conocimiento de $S D$ a través de $M$. Para alternativas ficcionalistas de esta relación véase Redmond y Valladares (2018a; 2018b) y Redmond, Valladares y Lopez-Orellana (2017).
}

Revista de Humanidades de Valparaíso, 2019, No 14, 315-334 
Un enfoque inferencial y dinámico de la modelización y de la comprensión en biología

Rodrigo Lopez-Orellana; Juan Redmond; David Cortés-García

(ii) $S$ genera hipótesis (inferencias subrogativas) sobre el $S D$ a partir de $M$.

El primero (i) es la relación que se establece por la capacidad de una fuente (que es $M)$ para llevar a un usuario competente e informado $(S)$ a considerar dicho sistema-diana $(S D)$. Se trata de una propiedad relacional y contextual de $M$, fijada y mantenida en parte por los usos previstos por parte de los agentes. No se puede decir que ningún modelo apunte intencionalmente a un sistema-diana en ausencia de tales usos. En este sentido, se trata de un acto intencional de $S$ que establece una relación entre $M$ y el $S D$.

También, si $M$ apunta a su sistema-diana, el requerimiento de direccionalidad queda garantizado ( $M$ apunta a $S D$ pero no al revés) y asegura al mismo tiempo (ii), esto es, la dinámica de inferencias subrogativas entre $M$ y $S D$.

Por razonamiento subrogativo o sustituto (surrogate reasoning) debe entenderse aquella función que cumple un modelo de generar hipótesis sobre su sistema-diana. Sustituto en el sentido de que las conclusiones que se obtengan por esta vía en $M$ serán consideradas como hipótesis formuladas sobre su sistema-diana. Lo que infiero en $M$ lo 'traslado' al sistema-diana.

Lo anterior nos permite definir inferencialmente una noción de comprensión que llamaremos aquí 'comprensión efectiva' (Lopez-Orellana y Cortés-García 2019):

El sujeto $S$ comprende efectivamente el modelo $M$, que habla acerca de un sistemadiana $S D$, cuando usa $M$ generando subrogativamente hipótesis que permiten manipular $S D$ mediante acciones o procesos de control y la generación de nuevos fenómenos $S D^{\prime}$.

Nuestra definición es entonces una definición inferencial y dinámica; 'comprensión efectiva' debe entenderse aquí como una noción de este tipo. Se ajusta a la definición de Elgin (citada más arriba) en la medida en que, para definir uso, nos focalizamos en las inferencias no triviales y argumentos que nos llevan a generar un conjunto de acciones o procesos para el control de los fenómenos. Y también, sobre todo en biología, para la generación de nuevos fenómenos. Afirmamos que esas acciones o procesos para el control de fenómenos (manipulación) tienen lugar en el experimento, como definiremos a continuación.

Así mismo, según la definición de comprensión de Elgin (2009), $M$ puede entenderse también como algún tipo de información (básica o muy elaborada o refinada) relativa a un fenómeno o sistema de la realidad $S D$, que en nuestro caso es la realidad biológica, y que puede ser transmitida o compartida con la comunidad científica. El éxito cognitivo de $S$ en la comprensión de $M$ (que habla acerca de $S D$ ), puede ser evaluado gracias a que logra manipular o usar satisfactoriamente $M$. En efecto, el éxito de la comprensión guarda relación, en última instancia, con el logro de $S$ en la transmisión o comunicación de $M$ y en la respectiva aceptación de sus pares. Con ello se sugiere que en dicho proceso cognitivo

Revista de Humanidades de Valparaíso, 2019, No 14, 315-334

(c) (1) (9) CC BY-NC-ND 
Un enfoque inferencial y dinámico de la modelización y de la comprensión en biología

Rodrigo Lopez-Orellana; Juan Redmond; David Cortés-García

el sujeto logra - en primera instancia - establecer relaciones y construir conocimientos efectivos y exitosos acerca de los hechos relativos a $S D$. De esta manera, se pone énfasis en el ámbito contextual y de aplicación de la comprensión. En el proceso de la comprensión, el científico usa o manipula la información en instancias donde necesite que sea más relevante, gracias a que ha 'captado' y logrado compartir su contenido. Entonces, una comprensión de este tipo debe ser entendida también como una cognición intersubjetiva acerca de un determinado fenómeno de estudio, que logra compartirse por el conjunto de la comunidad científica y que tiene como base epistémica la experimentación y la representación científicas asociadas al fenómeno.

Este modo de definir la comprensión en términos de uso, y el uso de los modelos a partir de su condición de generadores de hipótesis sobre $S D$, nos ubica en una clara perspectiva inferencialista y, por tanto, dinámica de la práctica de modelización en biología. Poner el peso en el uso aleja la modelización en biología de todo compromiso representacionalista, esto es, de una perspectiva estática de la modelización. No hay ningún compromiso realista: no se asume ningún isomorfismo o similaridad entre $M$ y $S D$.

\section{Noción de experimentación}

De acuerdo con nuestra definición de uso, "el científico usa un modelo cuando (i) apunta efectivamente con el modelo a un sistema-diana y (ii) genera hipótesis (inferencias sustitutas) sobre este sistema de fenómenos a partir del modelo", debe resultar claro el rol protagónico que posee la noción de experimento en nuestro enfoque, ya que hemos asumido que el ámbito primordial del uso de un modelo es el experimento. En este sentido, asumimos también que la noción de experimentación que está estrechamente relacionada con la comprensión es la de Ian Hacking (1983). En efecto, Hacking considera la experimentación como una práctica dinámica, como acción, basada en la intervención —o manipulación - y transformación de fenómenos, con cierta autonomía respecto de la(s) teoría(s) en cuestión ${ }^{6}$. Principalmente porque, al igual que con los modelos, la función de los experimentos no está reducida a la confirmación o refutación de esas teorías, aunque sí interactúa con la teorización y la modelización (Hacking 1983, 263-264).

En la concepción del realismo experimental (experimentalismo) de Hacking, realidad y experimentación están estrechamente vinculadas; en otras palabras, sostiene que la función principal de un experimento es crear fenómenos. Hacking habla de 'crear' y no de 'descubrir' atendiendo a la complejidad propia de la naturaleza, ya que generalmente es difícil tanto acceder a los fenómenos como producirlos de una manera estable y contro-

\footnotetext{
${ }^{6}$ Para Hacking, las relaciones entre teoría y experimento difieren en diferentes estadios de desarrollo, muchas veces los experimentos preceden a teorías importantes - como en el caso de las teorías de la óptica(Hacking, 1983, 159-160). Para una lectura complementaria sobre experimentación pueden verse Redmond y Lopez-Orellana (2018b) y Redmond y Valladares (2018b).
}

Revista de Humanidades de Valparaiso, 2019, No 14, 315-334

(c) $(1)(9)$ CC BY-NC-ND 
Un enfoque inferencial y dinámico de la modelización y de la comprensión en biología

Rodrigo Lopez-Orellana; Juan Redmond; David Cortés-García

lada. Con ello entendemos por qué es común para los científicos que sus experimentos fallen: "[i]gnorar este hecho es olvidar lo que la experimentación está haciendo [...] la verdadera destreza de los científicos es saber cuándo el experimento está funcionando" (Hacking 1983, 230).

Experimentar es entonces crear, producir, refinar y estabilizar fenómenos (Hacking 1983, 229-230). Es a través de esta práctica ordenada que el científico consigue el éxito en su investigación. Pero esto no quiere decir que Hacking asuma un antirrealismo científico acerca de las entidades o fenómenos del mundo. Según Hacking, el trabajo experimental proporciona la mejor evidencia para el realismo científico, no solo porque podamos poner a prueba las teorías acerca de esas entidades o fenómenos, sino más bien porque logramos manipular aquellas entidades que no pueden ser 'observadas' y porque logramos con ello producir nuevos fenómenos y comprender los aspectos de la naturaleza. Para Hacking, las entidades teóricas (como el electrón) o las relaciones fenoménicas supuestas por las teóricas (como la plasticidad fenotípica), son vistas en este enfoque como herramientas, es decir, se convierten en entidades experimentales o 'entidades para el experimentador' (Hacking 1983, 262-266).

En efecto, es esa manipulación la que nos permite acercarnos a una comprensión efectiva de los fenómenos de la realidad. Por ejemplo:

La entidad teórica favorita del filósofo es el electrón [...] los electrones se convierten en entidades experimentales, o en entidades para el experimentador. En los primeros estadios del descubrimiento de una entidad podemos poner a prueba la hipótesis de que existe. Incluso esto es rutina. Cuando J.J. Thomson se dio cuenta en 1897 de que lo que había llamado "corpúsculos" estaban saliendo de los cátodos calientes, casi la primera cosa que hizo fue medir la masa de estas partículas cargadas negativamente. Hizo una estimación cruda de $e$ y de la carga, y midió $e / m$. El valor que obtuvo de $m$ era bastante correcto. Millikan elaboró algunas de las ideas que ya se discutían en el laboratorio Cavendish, y en 1908 determinó la carga del electrón, esto es, la unidad mínima probable de carga eléctrica. Así, desde un principio, la gente ponía menos atención en probar la existencia del electrón que en el hecho de interactuar con ellos. Cuanto mejor sea nuestra comprensión acerca de los poderes causales del electrón, mejor podremos construir aparatos que logren efectos bien comprendidos en otras partes de la naturaleza. Cuando se logra usar el electrón para manipular otras partes de la naturaleza de una manera sistemática, el electrón deja de ser un ente hipotético o inferido. Ha dejado de ser teórico y se vuelve experimental. (Hacking 1983, 262, traducción nuestra)

En relación con esta perspectiva de Hacking, ya a principios del siglo XX, el biólogo Jacques Loeb había propuesto una epistemología específica para la biología basada en la idea del control y producción del conocimiento. Para Loeb, comprender un fenómeno es ser capaz de controlarlo y, por ende, de producirlo: 
Un enfoque inferencial y dinámico de la modelización y de la comprensión en biología

Rodrigo Lopez-Orellana; Juan Redmond; David Cortés-García

[...] la biología moderna es fundamentalmente una ciencia experimental y no descriptiva; [...] sus resultados no son retóricos, pero siempre asumen una de estas dos formas: o bien es posible controlar un fenómeno de la vida hasta el punto de que podamos producirlo a voluntad (como, v. g., la contracción de un músculo extirpado); o bien conseguimos encontrar la relación numérica entre las condiciones del experimento y el resultado biológico (v.g., la ley de herencia de Mendel). La biología, en la medida en que se basa en estos dos principios, no puede retroceder sino que debe avanzar. (Loeb 1912, 3-4)

En otras palabras, el objetivo primordial del biólogo es controlar o producir fenómenos, antes que representarlos. El conocimiento exitoso que nos ofrece la biología radica en esta cuestión. Loeb definía la biología como investigación centrada en el control de los organismos; de esta manera, la experimentación obviamente juega un papel central (Pauly 1987, 5). Toda justificación del conocimiento biológico viene dada por el éxito de ese control, y esto se hace a través de experimentos. Una prueba de ello, según Loeb, fue su desarrollo de la partenogénesis artificial (fertilización química o activación del óvulo) en 1899, con la cual se puede comprender cuáles fueron las condiciones para el desarrollo evolutivo del óvulo — entre otras cuestiones-. Este éxito le llevó a considerar y valorar la forma en la cual los científicos deben generar un conjunto de acciones o procesos para el control y la generación de nuevos fenómenos (Loeb 1903; 1904).

Ahora bien, este tipo de manipulación involucrada en la experimentación es distinta que en el caso de los modelos. En la experimentación el objeto de esa manipulación o intervención son los propios fenómenos. Como señalamos anteriormente, el ámbito de la manipulación directa de los modelos es el experimento, pero el objeto de esa manipulación es un modelo. No se debe confundir ambas instancias de manipulación, aunque por supuesto estas están relacionadas. Como lo muestran Morgan y Morrison (1999), los modelos en conjunto con los experimentos son los que actúan como efectivos mediadores entre los fenómenos y las teorías. Pero los modelos cumplen un rol primordial en cuanto a que uno de sus principales objetivos es la explicación de los fenómenos.

En efecto, y siguiendo a Cartwright et al. (1995, 138-139), ambos son instrumentos exitosos de investigación. Nos permiten - por un lado- intervenir en el dominio conceptual de interpretaciones y representaciones del mundo, y - por otro lado- nos permiten la intervención sobre el dominio fenoménico. Por ello, ambos elementos deben considerarse como las herramientas para la comprensión cientifica. Aunque la perspectiva de Hacking exagere el papel del experimento, es totalmente compatible con los enfoques de Morgan y Morrison (1999) y de Cartwright et al. (1995), en vistas de la noción de comprensión científica. Por supuesto, debemos reconocer que, a favor del experimentalismo de Hacking, los experimentos nos permiten poner a prueba las hipótesis subrogativas que puedan darse acerca de la existencia de los fenómenos en cuestión y — de esta maneranos blindan para no caer en un realismo ingenuo sobre esos fenómenos. 
Un enfoque inferencial y dinámico de la modelización y de la comprensión en biología

Rodrigo Lopez-Orellana; Juan Redmond; David Cortés-García

\section{Modelos metapoblacionales: comprensión e idealización matemática}

Por estas razones, afirmamos también que el éxito de la comprensión tiene una estrecha relación con el hecho de estar en posesión de un modelo. Generalmente, un modelo comporta en una primera instancia una estructura simple o básica de información, pero que puede ir mejorándose o refinándose a partir de la interpretación de los fenómenos o de los resultados de la experimentación en su uso o aplicación exitosa. Una forma de entender esto es poniendo atención sobre las clases de modelos que existen en biología y el grado de idealización que entrañan. Dar cuenta de la gran diversidad de modelos que se utilizan en biología - que se encuentra en estrecha relación con los usos de estos y con los objetivos epistémicos particulares de los investigadores - es muy relevante para la interpretación de las estructuras de información (o conocimiento). Los modelos en biología pueden ser clasificados de distintas maneras; pero aquí distinguimos dos clases de modelos atendiendo a su carácter:

(a) Modelos matemáticos. Aquellos modelos integrados parcial o totalmente por elementos matemáticos. Dentro de estos, podemos distinguirlos en relación con contenido de idealización que comportan:

(i) Modelos matemáticos ideales. Consisten en formulaciones matemáticas aplicadas a la biología, por lo que son modelos a priori, sin carga empírica (o con una carga empírica muy restringida). Son, en muchas ocasiones, construidos como modelos falsos. Por ejemplo: el modelo de competencia interespecífica de Lotka-Volterra, los modelos matriciales predictivos de sucesión ecológica, los modelos clásicos de genética poblacional y el modelo de biogeografía insular de McArthur y Wilson.

(ii) Modelos matemáticos empíricos. Son modelos que poseen una carga matemática importante, al incluir en su propia constitución funciones gráficas, ecuaciones, algoritmos, etc.; sin embargo, y a diferencia de los anteriores, parten de mediciones u observaciones empíricas. Pueden ser reconfigurados en modelos falsos. Por ejemplo: el modelo de la cinética enzimática de Michaelis-Menten, los modelos gráficos para los efectos Bohr y Haldane de la saturación de la hemoglobina y los modelos computacionales basados en la teoría de redes.

Esta distinción entre modelos ideales y modelos empíricos es una clasificación continua, establecida en base al grado de idealización que un modelo particular comporte, y siendo, generalmente, inversamente proporcional a su carga empírica. De esta manera, un modelo particular, en su desarrollo, puede alterar su grado de idealización y de carga empírica, como reflejaremos más adelante en esta misma sección.

Revista de Humanidades de Valparaíso, 2019, No 14, 315-334

(c) (1) $\odot$ CC BY-NC-ND 
Un enfoque inferencial y dinámico de la modelización y de la comprensión en biología

Rodrigo Lopez-Orellana; Juan Redmond; David Cortés-García

(b) Modelos descriptivos. Son de carácter fundamentalmente descriptivo. Por lo general son menos abstractos que los modelos matemáticos, aunque su elaboración comporta siempre un cierto grado de abstracción y de idealización, aunque no en un sentido matemático. Por ejemplo: los modelos de los operones lac y trp, el modelo de Singer de la bicapa lipídica, los modelos-organismo ${ }^{7}$ y los modelos $3 \mathrm{D}$ computacionales o físicos de estructuras anatómicas.

Incluso podemos encontrar diferentes modelos con distintos grados de idealización para un mismo sistema de fenómenos. Por ejemplo, para el caso de la predicción de la dinámica de metapoblaciones se han propuesto sucesivos modelos (Hanski 1998; Smith y Smith 2007, 255-269) que van incorporando progresivamente una carga empírica mayor, al tiempo que se reduce su grado de idealización y cuya 'precisión' representacional resulta incrementada. Ejemplos como estos permiten abordar el problema de los estándares de precisión representacional de los modelos y de la aplicabilidad de las matemáticas; esto es, el problema número (4) y la condición de suficiencia número (10) de Frigg y Nguyen (2016). En efecto, los modelos metapoblacionales tratan de predecir las variaciones a lo largo del tiempo en los tamaños de las subpoblaciones en casos de hábitat discontinuo, es decir, en poblaciones cuya distribución geográfica atiende a una conformación en parcelas. Aquellas poblaciones que se encuentras geográficamente fragmentadas, pero entre cuyas subpoblaciones se mantiene un flujo génico (migración) se denominan metapoblaciones. En la siguiente figura se representa un esquema de una metapoblación clásica con distribución parcelada:

\footnotetext{
${ }^{7}$ Denominamos modelo-organismo a aquellos modelos que se corresponden con un organismo biológico. El mismo individuo (o grupo de individuos) funcionan efectivamente como un modelo.
} 
Un enfoque inferencial y dinámico de la modelización y de la comprensión en biología

Rodrigo Lopez-Orellana; Juan Redmond; David Cortés-García

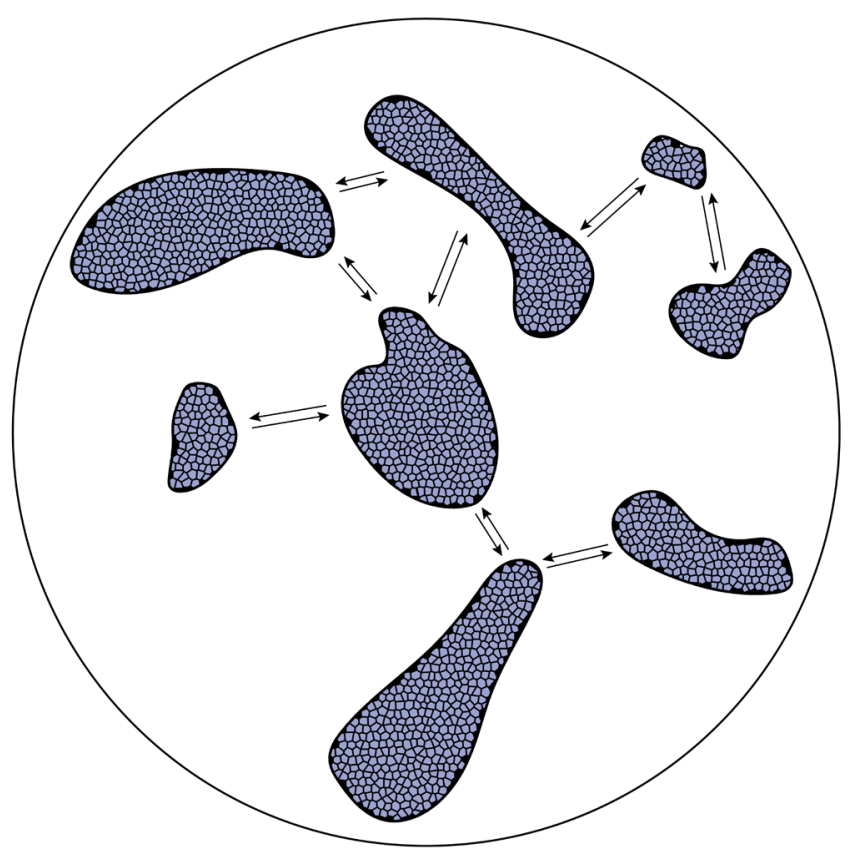

Fig. 1. Esquema de una metapoblación clásica con distribución parcelada. Los espacios coloreados (parcelas) representan el área de ocupación de una subpoblación, es decir, las regiones cuyas características bióticas y abióticas pueden mantener una subpoblación de la especie de interés, mientras que los espacios en blanco (matriz) es toda sección del terreno cuyas características las hacen inhabitables para una población determinada. Se corresponde con los espacios vacíos entre parcelas ocupadas. A diferencia de otros modelos metapoblacionales, el modelo clásico no distingue entre parcelas ocupadas y parcelas ocupables. Las flechas representan el flujo génico entre subpoblaciones, es decir, la migración entre parcelas.

De hecho, el modelo básico de metapoblaciones $\left(\mathbf{e}_{1}\right)$ pertenecería a la clase de modelos matemáticos ideales, mientras que podríamos considerar las sucesivas elaboraciones y refinamientos de este $\left(\mathbf{e}_{\mathbf{2}} \mathbf{y}_{\mathbf{3}} \mathbf{e}_{\mathbf{3}}\right)$ como modelos matemáticos empíricos. El modelo original parte de una serie de asunciones teóricas, consistiendo el mismo en la aplicación de una ecuación a un problema biológico real: las dinámicas demográficas en poblaciones geográficamente fracturadas. Su formulación matemática es la siguiente:

$$
\begin{array}{ll}
\mathbf{e}_{1} \quad \frac{d p}{d t}=I(p)-E(p) \quad \begin{array}{l}
\text { donde } p \text { es la probabilidad de persistencia (fracción de } \\
\text { espacios ocupados), } I(p) \text { la tasa de inmigración y } E(p) \text { la } \\
\text { tasa de extinción. }
\end{array}
\end{array}
$$


Un enfoque inferencial y dinámico de la modelización y de la comprensión en biología

Rodrigo Lopez-Orellana; Juan Redmond; David Cortés-García

Otra versión posterior es el modelo 'isla-continente' o 'lluvia de propágulos', que considera un núcleo mucho mayor en el que la población nunca llega a extinguirse (continente). Esta nueva formulación supone un refinamiento de la ecuación anterior al incorporar nuevas presuposiciones empíricas:

$$
\mathbf{e}_{2} \quad \frac{d p}{d t}=i(1-p)-e p \quad \begin{aligned}
& \text { donde } i \text { es la probabilidad de inmigración y } e \text { es la } \\
& \text { probabilidad de extinción. }
\end{aligned}
$$

Una versión que se ajusta mejor a las dinámicas poblacionales es el modelo de Levins, que considera que la tasa de inmigración no es constante sino proporcional a la fracción de parcelas ocupadas y a la fracción de parcelas vacías:

$$
\mathbf{e}_{3} \quad \frac{d p}{d t}=i(1-p) \cdot \mathrm{p}-e p
$$

Este es un modelo mucho más simple. Nos permite comprender la relación entre la tasa de colonización de parcelas y la tasa de extinción de subpoblaciones. Ambos casos dependen de las características observadas de las especies, específicamente, de la habilidad de las especies para migrar: cuanto más hábil sea una especie para migrar, mayor será la tasa de ocupación (colonización) de una parcela; y, cuanto mayor sea la tasa de extinción, mayor será la tasa de desocupación (Levins 1969; 1970).

A pesar de que el modelo de Levins $\left(\mathbf{e}_{3}\right)$ se aproxime mejor a la dinámica poblacional observada en la naturaleza, cabe señalar que no tiene en consideración otros aspectos relevantes tales como el efecto rescate, las características de la matriz, la importancia de los corredores ecológicos ${ }^{8}$, los factores ambientales regionales, la no-linearidad de las relaciones en la naturaleza, las sinergias causales, los efectos antropogénicos, etc. (Laurance 2008). La incorporación de estos factores abre la posibilidad de elaborar modelos de mayor precisión representacional para casos experimentales particulares, atendiendo a las características propias la metapoblación concreta a modelar (incluyendo las características del entorno) y de los objetivos epistémicos particulares de los investigadores. Esto nos muestra que, en muchos casos, los modelos ideales difícilmente son capaces de capturar la complejidad de los fenómenos que tratan de explicar o predecir, principalmente porque en esta ciencia predominan las causas multifactoriales. Aunque debemos destacar que el valor de los modelos metapoblacionales viene dado por su poder predictivo, puesto que estos son elaborados con el fin de comprender las dinámicas poblacionales en hábitats fragmentados para desarrollar programas de gestión del territorio y de conservación de la biodiversidad más eficientes.

\footnotetext{
${ }^{8}$ Los corredores ecológicos son fracciones de terreno que unen diferentes parcelas y que, no formando parte del espacio habitable, favorecen el movimiento de los individuos entre parcelas y, por lo tanto, el flujo génico.
} 
Un enfoque inferencial y dinámico de la modelización y de la comprensión en biología

Rodrigo Lopez-Orellana; Juan Redmond; David Cortés-García

\section{Conclusiones}

Con el ejemplo anterior, el modelo metapoblacional de Levins, podemos confirmar que el éxito asociado a un modelo particular viene dado por su uso. En efecto, el incremento de la carga empírica asociada a un modelo particular - fruto de la incorporación de nuevos elementos causales, factores, o variables - supone un aumento en la precisión de la representación (ejemplificada en el paso de $\mathbf{e}_{\mathbf{1}}$ a $\mathbf{e}_{3}$ ). Esto supone la obtención de una mejor comprensión de los fenómenos involucrados, ya que nos permite inferir mejores hipótesis acerca de los mismos. De esta manera, podemos afirmar que el poder predictivo de un modelo viene dado por el carácter inferencial de la comprensión que nos ofrece.

En efecto, si enfocamos esta cuestión a nuestra definición de comprensión efectiva, podemos reconocer que un sujeto $S$ usa el modelo $M$ (por ejemplo, $\mathbf{e}_{1}$ o $\mathbf{e}_{2}$ ) generando hipótesis que permiten manipular $S D$ (en nuestro ejemplo, un conjunto de poblaciones próximas que intercambian individuos de la misma especie), mediante acciones o procesos de control (por ejemplo, manipulando genéticamente a un grupo de sus individuos o interviniendo en su ecosistema ${ }^{9}$ ). Estas acciones y procesos de control están alineados con las predicciones basadas en las inferencias realizadas subrogativamente a partir de $M$ y el correspondiente testeo en la experiencia. Este testeo confirma las predicciones aumentando la comprensión efectiva y permitiendo un margen de mejora en el que aumenta la capacidad predictiva. Todo esto se traducirá en la obtención de un mejor modelo $M^{\prime}$ (en nuestro ejemplo, gracias a la inclusión de la tasa de inmigración proporcional en $\mathbf{e}_{3}$ ).

De este modo, podemos ver también que la noción de predicción, como conjunto inferencias exitosas, nos permite pensar en una versión dinámica de la comprensión científica. En efecto, se trataría un proceso que nos ayuda a explicar cómo el modelo va mejorando. Incluso, podemos decir que el modelo como representación científica es más preciso o adecuado solo a la luz de las inferencias exitosas que, como predicciones, son el resultado de una comprensión efectiva que un usuario generó al usar el modelo prediciendo y realizando acciones con éxito sobre su sistema-diana.

Con todo esto, podemos ver el importante rol epistémico que tiene la comprensión para la explicación con modelos en biología, esto es, definida inferencial y dinámicamente como comprensión efectiva.

\footnotetext{
${ }^{9}$ Para un ejemplo exhaustivo de manipulación de un sistema de fenómenos sobre metapoblaciones, véase el famoso experimento de Klassen et al. (1970). Se trata de la manipulación de los caracteres hereditarios de una especie, como la Anthonomus grandis o gorgojo del algodón, para generar rasgos condicionales letales con el fin de destruir un porcentaje importante de individuos de esa especie y medir su respuesta de sobrevivencia global.
}

Revista de Humanidades de Valparaíso, 2019, No 14, 315-334 
Un enfoque inferencial y dinámico de la modelización y de la comprensión en biología Rodrigo Lopez-Orellana; Juan Redmond; David Cortés-García

\section{Referencias bibliográficas}

Braillard, P.-A., Malaterre, C. (2015). Explanation in Biology: An Introduction. En P.-A. Braillard, C. Malaterre (eds.), Explanation in Biology. An Enquiry into the Diversity of Explanatory Patterns in the Life Sciences, Vol. 11, pp. 1-28. Dordrecht: Springer. doi: https://doi. org/10.1007/978-94-017-9822-8

Cartwright, N., Shomar, T., Suárez, M. (1995). The Tool Box of Science. Tools for the Building of Models with a Superconductivity Example. En W. E. Herfel, W. Krajewski, I. Niiniluoto, R. Wójcicki (eds.), Theories and Models in Scientific Processes, pp. 138-149. Amsterdam: Editions Rodopi B.V.

Cassini, A. (2016). Modelos científicos. Diccionario Interdisciplinar Austral. Retrieved from: http://dia.austral.edu.ar/Modelos

Darwin, C. (1859). On the Origin of Species by Means of Natural Selection, or, The Preservation of Favoured Races in the Struggle for Life. London: John Murray.

de Regt, H. W. (2009). Understanding and scientific explanation. En H. W. de Regt, S. Leonelli, K. Eigner (eds.), Scientific Understanding. Philosophical Perspectives, pp. 21-42. Pittsburgh: University of Pittsburgh Press.

de Regt, H. W., Dieks, D. (2005). A contextual approach to scientific understanding. Synthese, 144(1): 13-170. doi: https://doi.org/10.1007/s11229-005-5000-4

de Regt, H. W., Leonelli, S., Eigner, K. (2009). Focusing on scientific understanding. En H. W. de Regt, S. Leonelli, K. Eigner (eds.), Scientific Understanding. Philosophical Perspectives, pp. 1-17. Pittsburgh: University of Pittsburgh Press.

Elgin, C. Z. (2009). Is Understanding Factive? In A. Haddock, A. Millar, D. Pritchard (eds.), Epistemic Value, pp. 322-330. Oxford University Press.

Frigg, R., Hartmann, S. (2018). Models in Science. The Stanford Encyclopedia of Philosophy. Retrieved from: https://plato.stanford.edu/archives/sum2018/entries/models-science/

Frigg, R., Nguyen, J. (2016). Scientific Representation. The Stanford Encyclopedia of Philosophy. Retrieved from: https://plato.stanford.edu/archives/win2018/entries/scientificrepresentation

Hacking, I. (1983). Representing and intervening. Introductory topics in the philosophy of natural science. Cambridge: Cambridge University Press.

Hanski, I. (1998). Metapopulation dynamics. Nature, 396(6706): 41-49. https://doi. org/10.1016/0169-5347(89)90061-X

Kitcher, P. (1981). Explanatory Unification. Philosophy of Science, 48(4): 507-531.

Kitcher, P. (1989). Explanatory Unification and the Causal Structure of the World. En P. Kitcher, W. Salmon (eds.), Scientific Explanation, pp. 410-505. Minneapolis: University of Minnesota Press. 
Un enfoque inferencial y dinámico de la modelización y de la comprensión en biología

Rodrigo Lopez-Orellana; Juan Redmond; David Cortés-García

Klassen, W., Creech, J. F., Bell, A. (1970). The potential for genetic suppression of insect populations by their adaptations to climate. Miscellaneous Publication, USDA, 1178: 1-77.

Knuuttila, T., Merz, M. (2009). Understanding by Modeling An Objectual Approach. En H. W. de Regt, S. Leonelli, K. Eigner (eds.), Scientific Understanding. Philosophical Perspectives, pp. 146-168. Pittsburgh: University of Pittsburgh Press.

Laurance, W. F. (2008). Theory meets reality: How habitat fragmentation research has transcended island biogeographic theory. Biological Conservation, 141(7): 1731-1744. https://doi. org/10.1016/j.biocon.2008.05.011

Leonelli, S. (2009). Understanding in Biology: The Impure Nature of Biological Knowledge. En H. W. de Regt, S. Leonelli, K. Eigner (eds.), Scientific Understanding. Philosophical Perspectives, pp. 189-209. Pittsburgh: University of Pittsburgh Press.

Levins, R. (1969). Some demographic and genetic consequences of environmental heterogeneity for biological control. Bulletin of the Entomological Society of America, 15(3): 237-240. doi: https://doi.org/10.1093/besa/15.3.237

Levins, R. (1970). Extinction. En M. Desternhaber (ed.), Some mathematical problems in biology. Lectures on Mathematics in the Life Sciences, Vol. 1, pp. 77-107. Providence, Rhode Island: American Mathematical Society.

Loeb, J. (1912). The Mechanistic Conception of Life. Biological Essays. Chicago: The University of Chicago Press.

Lopez-Orellana, R., Cortés-García, D. (2019). On Understanding and Modeling in Evo-Devo. An Analysis of the Polypterus Model of Phenotypic Plasticity. In A. Nepomuceno, L. Magnani, F. Salguero, C. Barés and M. Fontaine (eds.), Model-Based Reasoning in Science and Technology. Inferential Models for Logic, Language, Cognition and Computation, pp. 138-152. SAPERE Series. Cham: Springer. doi: https://doi.org/10.1007/978-3-030-327224_9

Lorenzano, P. (2008). Lo a priori constitutivo en la ciencia y las leyes (y teorías) científicas. Revista de Filosofia, 33(2): 21-48.

Mäki, U. (2009). MISSing the world. Models as isolations and credible surrogate systems. Erkenn, 70: 29-43. doi:10.1007/s10670-008-9135-9

Morrison, M., Morgan, M. S. (1999). Models as Mediating Instruments. En Mary S. Morgan and Margaret Morrison (eds.), Models as Mediators. Perspectives on Natural and Social Science, pp. 10-37. Cambridge: Cambridge University Press.

Newman, M. P. (2017). Theoretical understanding in science. British Journal for the Philosophy of Science, 68(2): 571-595. doi: https://doi.org/10.1093/bjps/axv041

Pauly, P. J. (1987). Controlling Life. Jacques Loeb and the Engineering Ideal in Biology. Oxford: Oxford University Press.

Redmond, J., Lopez-Orellana, R. (2018a). Lógica clásica y esquizofrenia: por una semántica lúdica. Revista de Filosofia, 74: 197-223. doi: 10.4067/S0718-43602018000100215. 
Un enfoque inferencial y dinámico de la modelización y de la comprensión en biología

Rodrigo Lopez-Orellana; Juan Redmond; David Cortés-García

Redmond, J., Lopez-Orellana, R. (2018b). Revisando las prácticas científicas de Galileo. En D. Salatino, G. Cuadrado y L. Gómez (eds.), Creatividad, investigación y lógica transcursiva, pp. 345-354. Mendoza: Facultad Regional Mendoza Universidad Tecnológica Nacional.

Redmond, J., Valladares, D. (2018a). Ficción en ciencia. Por una lectura artefactual de los modelos en ciencia. En Olga Pombo (ed.), Modelos e Imagens, pp. 45-73. Lisboa: Fim de Século Edições.

Redmond, J., Valladares, D. (2018b). Revisiting the Bohr Model from an artefactual perspective. En O. Pombo, A. Pato y J. Redmond (eds.), Epistemologia, Lógica e Linguagem, pp. 85106. Colecção Documenta. Lisboa: CFCUL.

Redmond, J., Valladares, D. L., Lopez-Orellana, R. (2017). Modelizaciones galileanas y objetos ideales. En Guillermo Cuadrado y L. E. Gómez (eds.), Ciencias de la Ingeniería en el Siglo XXI. Nuevos enfoques en su lógica, enseñanza y práctica, pp. 51-61. Mendoza: Universidad Tecnológica Nacional.

Santesmases, M. J. (2002). ¿Artificio o naturaleza? Los experimentos en la historia de la biología. Theoria, 17(2): 265-289.

Smith, T. M., Smith, R. L. (2007). Ecología. Madrid: Pearson Educación.

Suárez, M. (2003). Scientific representation: against similarity and isomorphism. International Studies in the Philosophy of Science, 17(3): 225-244.

Suárez, M. (2004). An inferential conception of scientific representation. Philosophy of Science, 71(5): 767-779.

Wilkenfeld, D. A. (2013). Understanding as representation manipulability. Synthese, 190(6): 9971016. doi: https://doi.org/10.1007/s11229-011-0055-x

Woodward, J. (2013). Making Things Happen: A Theory of Causal Explanation. New York: Oxford University Press. 